\title{
QUERUBISMO: RELATO DE CASO E REVISÃO DA LITERATURA COM ASPECTOS IMAGINOLÓGICOS*
}

\author{
Tarcísio Nunes Carvalho ${ }^{1}$, Cyrillo Rodrigues de Araújo Júnior ${ }^{1}$, Marlos Augusto Bittencourt \\ Costa ${ }^{1}$, Humberto de Souza Pereira Barcelos ${ }^{1}$, Tanise Nunes Carvalho² ${ }^{2}$ Carlos Alberto Ximenes ${ }^{3}$, \\ Kim-Ir-Sen Santos Teixeira ${ }^{4}$
}

\begin{abstract}
Resumo Querubismo é uma doença óssea hereditária não neoplásica caracterizada, clinicamente, por aumento indolor bilateral da mandíbula e maxilar em crianças, produzindo uma aparência querubínica. Pode ocorrer em casos isolados ou em membros de uma mesma família. Relatamos o caso de querubismo em uma menina sem história familiar, com lesões osteolíticas expansivas na mandíbula e maxila demonstradas em exames radiológicos.
\end{abstract}

Unitermos: Querubismo; Doenças mandibulares; Radiologia; Tomografia computadorizada.

Abstract Cherubism: case report and review of the literature and imaging findings.

Cherubim is a non-neoplastic hereditary bone disease characterized by bilateral, painless enlargements of the mandible and maxilla in children, producing the cherubic appearance. Cherubism may appear as an isolated case or in members of the same family. We report the case of a girl without familial history of cherubism who presented expansile osteolytic lesions in the mandible and maxilla, demonstrated by radiological exams. Key words: Cherubism; Mandibular diseases; Radiology; Computed tomography.

\section{INTRODUÇÃO}

Querubismo é uma lesão fibro-óssea hereditária não neoplásica, histologicamente semelhante ao granuloma de célula gigante da mandíbula, que compromete a mandíbula e a maxila de crianças, bilateral e simetricamente, produzindo uma aparência querubínica ${ }^{(1)}$.

A doença foi descrita primeiro em 1933 por Jones ${ }^{(2)}$ e denominada de doença multilocular familiar da mandíbula, porém depois que a sua natureza cística foi invalidada, Jones et al. ${ }^{(3)}$ foram os primeiros a usar o termo "querubismo". Este é inapropriado, visto que as crianças com esta enfermidade apresentam-se com as características faciais de um anjo barroco e não de um querubim clássico, com a face infantil de bochechas cheias ${ }^{(4)}$ (Figura1).

\footnotetext{
* Trabalho realizado no Hospital das Clínicas da Faculdade de Medicina da Universidade Federal de Goiás (FM-UFG), Goiânia, GO.

1. Médicos Residentes do Serviço de Radiologia e Diagnós tico por Imagem do Hospital das Clínicas da FM-UFG.

2. Acadêmica do Curso de Medicina da FM-UFG.

3. Professor Adjunto do Serviço de Radiologia e Diagnóstico por Imagem do Hospital das Clínicas da FM-UFG.

4. Professor Doutor do Serviço de Radiologia e Diagnóstico por Imagem do Hospital das Clínicas da FM-UFG.

Endereço para correspondência: Dr. Tarcísio Nunes Carvalho. Rua C-167, no 130, L-15, Q-588, Setor Nova Suíça. Goiânia, GO, 74255-100. E-mail: tarcisionunes@hotmail.com

Recebido para publicação em 11/8/2003. Aceito, após revisão, em 10/9/2003.
}

É uma doença benigna rara, com herança autossômica dominante. Uma variedade de outros nomes foi usada para descrever esta condição, incluindo displasia fibrosa hereditária ou familiar, tumor de célula gigante bilateral e doença multilocular familiar $^{(4)}$. Aparentemente há uma penetrância de $100 \%$ em meninos e somente $50 \%$ a $70 \%$ em meninas ${ }^{(5)}$.

A avaliação histopatológica evidencia proliferação do tecido conjuntivo fibroso contendo numerosas células gigantes multinucleadas ${ }^{(4)}$.

O diagnóstico de querubismo é realizado pelo exame histopatológico e, principalmente, pela evolução clínica, história familiar e achados radiológicos ${ }^{(6)}$.

Desde a sua primeira descrição em 1933, cerca de 200 casos de querubismo foram relatados na literatura ${ }^{(4)}$.

\section{RELATO DO CASO}

Paciente do sexo feminino, nove anos de idade, manifestou aumento progressivo e indolor das bochechas, iniciado há cerca de sete anos. Atualmente refere má oclusão dentária e ansiedade devido à sua deformidade facial.

O exame físico evidenciou deformidade facial caracterizada por aumento bilateral e assimétrico das bochechas e infra-orbi-

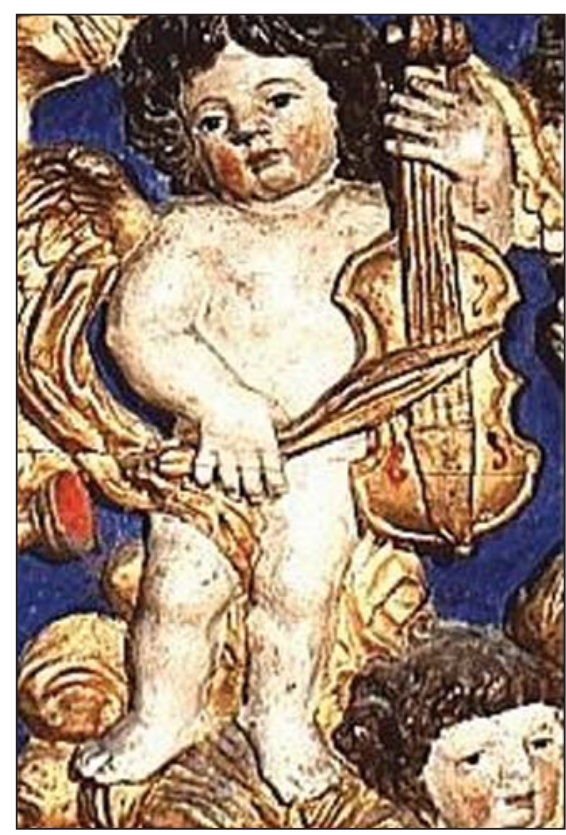

Figura 1. Anjos barrocos de típica face infantil, com as bochechas salientes.

tal, mais acentuada à direita (Figura 2). Notava-se ainda elevação do globo ocular direito em relação ao lado contralateral. Não havia perda visual ou alteração de movimentos oculares. Não foram observados casos semelhantes na família.

A radiografia demonstrou lesões osteolíticas insuflativas nos ângulos e ramos mandibulares. Não havia evidência de 


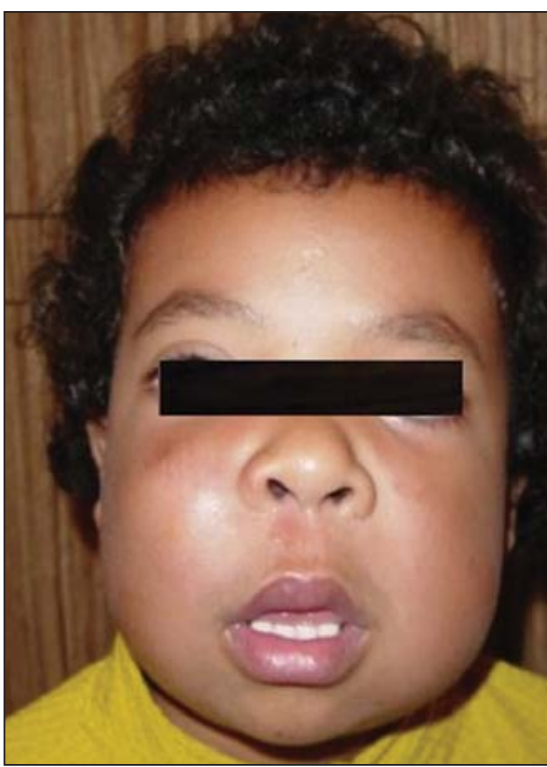

Figura 2. Fotografia da paciente demonstrando a deformidade facial (aparência "querubínica").

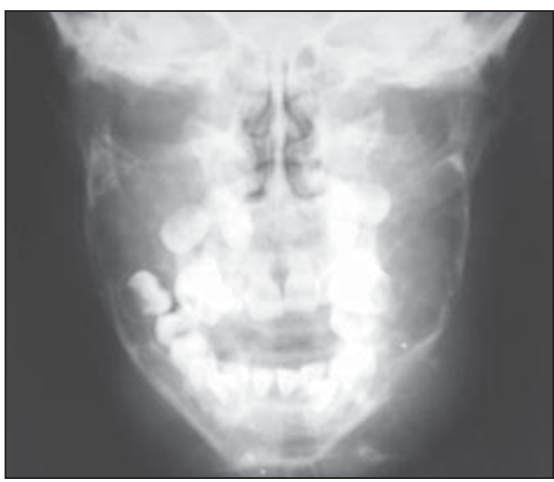

Figura 3. Radiografia. Lesões osteolíticas insuflativas nos ângulos e ramos mandibulares: aspecto de "dentes flutuantes".

pneumatização dos seios maxilares. As lesões maxilares eram mal caracterizadas neste exame (Figura 3).

A tomografia computadorizada (TC) caracterizou-se por lesões osteolíticas insuflativas, sólidas, bilateralmente, sendo simétricas nos ângulos e ramos ascendentes da mandíbula e assimétricas nos ossos maxilares, com acometimento mais extenso à direita, notando-se conseqüente elevação do assoalho da órbita e ausência de pneumatização do seio maxilar ipsilateral. O seio maxilar esquerdo estava discretamente pneumatizado (Figura 4).

Realizou-se biópsia da lesão maxilar e o exame histopatológico revelou presença de múltiplas células gigantes no interior de tecido celular fibroso (Figura 5).
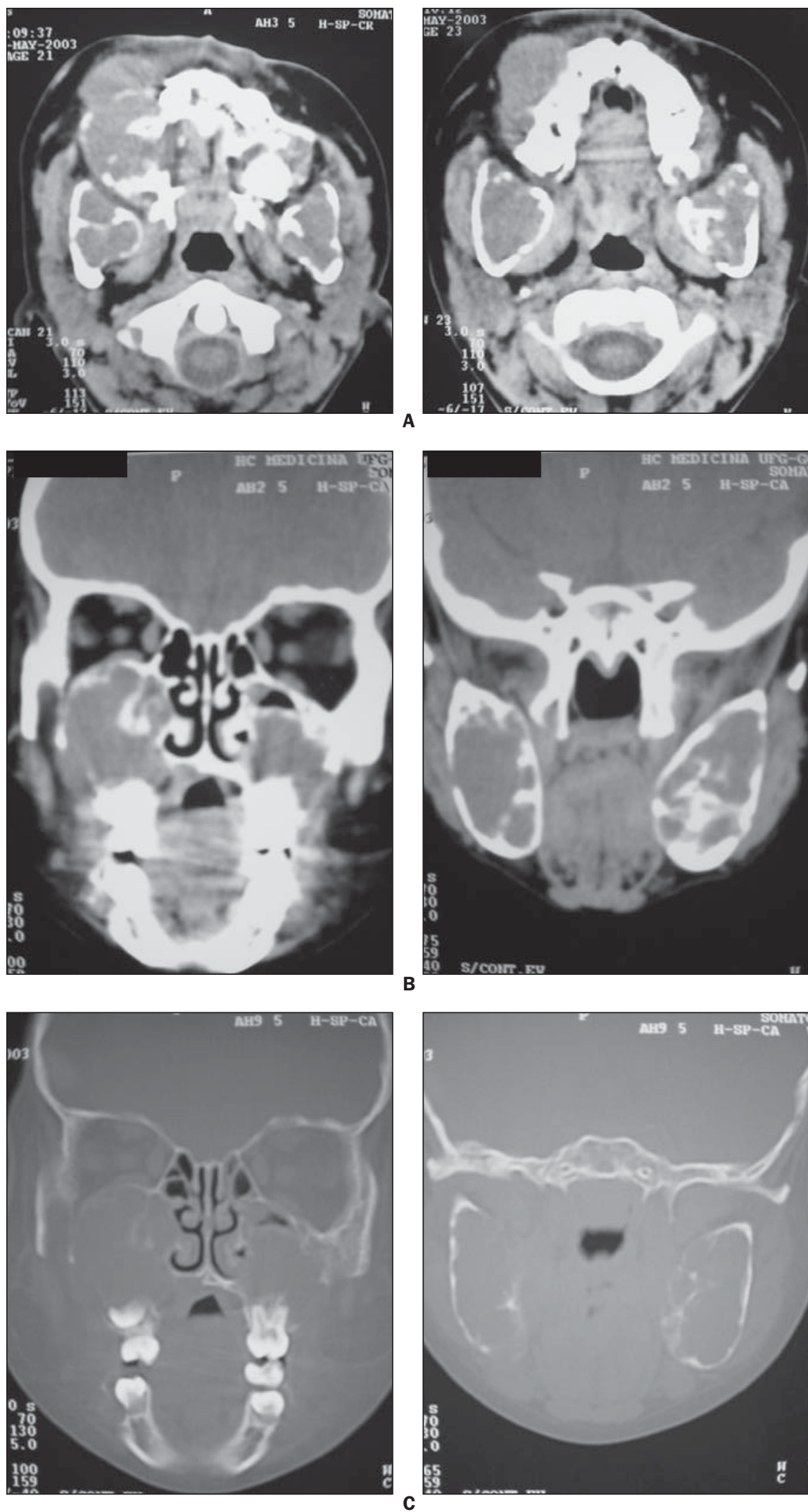

Figura 4. Tomografia computadorizada em planos axial (A), coronal (B) e coronal com janela óssea (C). Lesões osteolíticas insuflativas sólidas, simétricas nos ângulos e ramos ascendentes mandibulares e assimétricas nos ossos maxilares, com acometimento mais acentuado à direita, associadas a elevação do assoalho da órbita. 

Tecido conjuntivo fibroso contendo numerosas células gigantes multinucleadas. Nota-se ainda fragmento ósseo no material biopsiado.
Figura 5. Exame histopatológico.

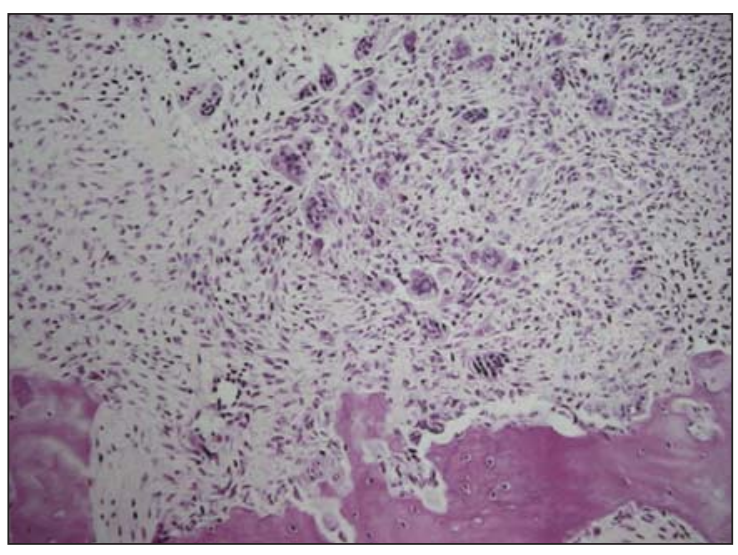

\section{DISCUSSÃO}

As crianças acometidas são normais ao nascimento e permanecem sem alterações clínicas e radiológicas até 14 meses a três anos de idade, quando se inicia o aumento simétrico mandibular. O crescimento ósseo torna-se autolimitado numa idade de cinco anos e cessa de 12 a 15 anos. Na puberdade as lesões começam a regredir. O remodelamento mandibular permanece pela terceira década de vida e ao final desta a anormalidade clínica pode ser sutil ${ }^{(5)}$. Os sinais e sintomas dependem da gravidade desta condição, variando de clínico ou radiologicamente sutil até uma deformidade acentuada acometendo a mandíbula e maxila, podendo determinar obstrução respiratória e perda visual ${ }^{(5)}$.

$\mathrm{O}$ querubismo foi inicialmente descrito como uma doença familiar acometendo a mandíbula, porém casos isolados sem origem hereditária têm sido relatados ${ }^{(4)}$.

Arnott ${ }^{(7)}$ sugeriu um sistema de graduação progressiva para as lesões do querubismo: grau I, caracterizado por envolvimento de ambos os ramos ascendentes mandibulares; grau II, por envolvimento de ambas as tuberosidades maxilares, assim como ramos ascendentes mandibulares; grau III, por acometimento extenso de toda a maxila e mandíbula, exceto processos coronóides e côndilos. $\mathrm{O}$ acometimento maxilar pode ser assimétrico.

Radiologicamente o querubismo caracteriza-se por lesões osteolíticas insuflativas, bilateralmente, na mandíbula. Lesões precoces ocorrem no corpo posterior e no ramo ascendente mandibular. As lesões maxilares podem ocorrer ao mesmo tem- po e às vezes não são identificadas precocemente no exame radiográfico devido à sobreposição óssea nesta região. A destruição da cavidade alveolar pode deslocar os dentes, produzindo um aspecto radiográfico definido como "dentes flutuantes"(8). As lesões maxilares são pouco caracterizadas no exame radiográfico, notando-se apenas uma hipopneumatização do antro maxilar, porém a TC evidencia melhor estas lesões ósseas determinando o grau de redução do antro maxilar e da elevação do assoalho da órbita. A TC, contudo, demonstra lesões precoces na mandíbula e maxila bilateralmente, o grau de acometimento pela doença e a natureza sólida destas lesões ósseas ${ }^{(9)}$. O plano coronal da TC também pode evidenciar a redução vertical das dimensões da órbita e o grau de compressão do nervo óptico ${ }^{(10)}$. Na adolescência as áreas líticas começam a reossificar, resultando em esclerose irregular ${ }^{(4)}$.

O exame histopatológico revela proliferação do tecido conjuntivo fibroso contendo numerosas células gigantes multinucleadas. As lesões mais antigas demonstram aumento de tecido fibroso, diminuição do número de células gigantes e neoformação óssea. Os achados microscópios raramente permitem um diagnóstico específico de querubismo na ausência da informação clínica e radiológica ${ }^{(11)}$.

Laskin $^{(12)}$ referiu que "o tratamento do querubismo deveria ser baseado no conhecimento da evolução natural da doença e no comportamento clínico de cada caso individualmente". A cirurgia para correção de deformidades, portanto, é raramente indicada. Se necessária, a cirurgia é geralmente realizada após a puberdade, quando as lesões são autolimitadas, porém considerações estéticas ou problemas funcionais graves podem justificar o tratamento precoce $^{(1)}$. Radioterapia tem sido realizada com sucesso, porém é desencorajada pela possibilidade de retardo no crescimento da mandíbula, assim como osteo-radionecrose e indução de malignidade. Outros tratamentos, como curetagem e uso de calcitonina, também têm sido realizados ${ }^{(5)}$.

Este caso apresenta evolução clínica e achados radiológicos típicos do querubismo. A TC mostra o extenso acometimento maxilar e mandibular. O diagnóstico baseou-se na faixa etária, na evolução clínica e nos achados imaginológicos e histopatológicos. Não há história familiar, porém esta condição pode apresentar-se em meninas com querubismo, já que a penetrância neste sexo é de somente $50 \%$ a $70 \%$.

Este artigo tem o objetivo de relatar um caso não familiar de querubismo, além de realizar revisão da literatura, dando ênfase aos aspectos clínicos e imaginológicos essenciais no diagnóstico específico, visto ser o exame histopatológico isoladamente insuficiente.

\section{REFERÊNCIAS}

1. Timosca GC, Galesanu RM, Cotutiu C, Grigoras M. Aggressive form of cherubism: report of a case. J Oral Maxillofac Surg 2000;58:336-44.

2. Jones WA. Familial multilocular cystic disease of the jaws. Am J Cancer 1933;17:946-50.

3. Jones WA, Gerrie J, Pritchard J. Cherubism - a familial fibrous dysplasia of the jaws. Oral Surg Oral Med Oral Pathol 1952;5:292-5.

4. Ongole R, Pillai RS, Pai KM. Cherubism in siblings: a case report. J Can Dent Assoc 2003;69: 150-4.

5. Lannon DA, Earley MJ. Cherubism and its charlatans. Br J Plast Surg 2001;54:708-11.

6. Carroll AL, Sullivan TJ. Orbital involvement in cherubism. Clin Experiment Ophthalmol 2001;29: 38-40.

7. Arnott DG. Cherubism - an initial unilateral presentation. Br J Oral Surg 1978;16:38-46.

8. Caballero R, Vinals H. Cherubism: a study of three generations. Med Oral 1998;3:163-71.

9. Bianchi SD, Boccardi A, Mela F, Romagnoli R. The computed tomographic appearances of cherubism. Skeletal Radiol 1987;16:6-10.

10. Ahmadi AJ, Pirinjian GE, Sires BS. Optic neuropathy and macular chorioretinal folds caused by orbital cherubism. Arch Ophthalmol 2003;121: 570-3.

11. Neville BW, Damm DD, Allen CM, Bouquot JE Oral and maxillofacial pathology. 2nd ed. Philadelphia, PA: WB Saunders, 2002:547-9.

12. Laskin DM. Oral and maxillofacial surgery. St. Louis, MO: CV Mosby, 1985:585-91. 